\title{
Low-Voltage, Flexible IGZO Transistors Gated by PSSNa Electrolyte
}

DOI:

10.1109/LED.2018.2862910

10.1109/LED.2018.2862910

\section{Document Version}

Accepted author manuscript

Link to publication record in Manchester Research Explorer

\section{Citation for published version (APA):}

Du, L., He, D., Liu, Y., Xu, M., Wang, Q., Xin, Q., \& Song, A. (2018). Low-Voltage, Flexible IGZO Transistors Gated by PSSNa Electrolyte. IEEE Electron Device Letters, 39(9), 1334 - 1337.

https://doi.org/10.1109/LED.2018.2862910, https://doi.org/10.1109/LED.2018.2862910

\section{Published in:}

IEEE Electron Device Letters

\section{Citing this paper}

Please note that where the full-text provided on Manchester Research Explorer is the Author Accepted Manuscript or Proof version this may differ from the final Published version. If citing, it is advised that you check and use the publisher's definitive version.

\section{General rights}

Copyright and moral rights for the publications made accessible in the Research Explorer are retained by the authors and/or other copyright owners and it is a condition of accessing publications that users recognise and abide by the legal requirements associated with these rights.

\section{Takedown policy}

If you believe that this document breaches copyright please refer to the University of Manchester's Takedown Procedures [http://man.ac.uk/04Y6Bo] or contact uml.scholarlycommunications@manchester.ac.uk providing relevant details, so we can investigate your claim.

\section{OPEN ACCESS}




\title{
Low-Voltage, Flexible IGZO Transistors Gated by PSSNa Electrolyte
}

\author{
Lulu Du, Dandan He, Yaxuan Liu, Mingsheng Xu, Qingpu Wang, Qian Xin, and Aimin Song, senior \\ Member, IEEE
}

\begin{abstract}
A carving, cutting, and flip-chip bonding process is proposed for fabrication of flexible electric double layer transistors (EDLTs) with low cost. Solution processed poly(styrenesulfonic acid sodium salt) (PSSNa) is used as gate dielectric. The large EDL-specific capacitance $\left(4.5 \mu \mathrm{F} / \mathrm{cm}^{2}\right.$ at 20 Hz) can induce very high charge carrier density in the InGaZnO (IGZO) channel layer, enabling the EDLTs to operate at a single-battery-drivable low voltage of $1.0 \mathrm{~V}$ with a high on-current of $>10^{-4} \mathrm{~A}$. The effect of IGZO layer thickness on the performance of EDLTs was investigated. The flexible EDLT with optimized IGZO thickness of $100 \mathrm{~nm}$ has achieved a high on/off ratio of $1.4 \times$ $10^{7}$, a low threshold voltage of $0.51 \mathrm{~V}$, a saturated field-effect mobility of $1.14 \mathrm{~cm}^{2} / \mathrm{Vs}$, and high positive gate bias stress stability. Furthermore, the achieved subthreshold swing, $76 \mathrm{mV} / \mathrm{dec}$, is very close to the theoretical ideal minimum value.
\end{abstract}

Index Terms-Electric double layer transistors (EDLTs); Carving, cutting, and flip-chip bonding (CCFB); Flexible; Low operating voltages.

\section{INTRODUCTION}

$\mathrm{A}$ MORPHOUS oxide semiconductors, represented by InGaZnO (IGZO), are considered as one of the most promising semiconductors for flexible thin film transistors (TFTs) due to their high mobility $\left(\sim 1-100 \mathrm{~cm}^{2} / \mathrm{Vs}\right)$, visible light transparency, low-temperature (even room-temperature) and large-area processability, and excellent mechanical flexibility [1]-[3]. Flexible and transparent TFTs based on amorphous oxide semiconductors have great application prospects in the next generation wearable and portable electronics [4]-[6]. At present, a major challenge of battery-powered wearable or portable electronics is to reduce the operating voltage and power consumption [7]. Electric double layer (EDL) transistors (EDLTs) are promising candidates for low-voltage operations

The authors thank J. Du for his guidance on cutting technology. This work was supported in part by the National Key Research and Development Program of China (Grant No. 2016YFA0301200 and 2016 YFA0201800), Engineering and Physical Sciences Research Council (EPSRC) (Grant No. EP/N021258/1), China Postdoctoral Science Foundation funded project (2016M590634), the Key Research and Development Program of Shandong Province (2017GGX10111 and 2017GGX10121), the Natural Science Foundation of Shandong Province (ZR2018MF029), the Natural Science Foundation of Jiangsu Province (BK20151255), and the Fundamental Research Funds of Shandong University (2016WLJH44).

L. Du, D. He, Y. Liu, M. Xu, Q. Wang, Q. Xin, and A. Song are with Center of Nanoelectronics, State Key Laboratory of Crystal Materials, and School of Microelectronics, Shandong University, Jinan 250100, P. R. China (e-mail: xinq@sdu.edu.cn).

A. Song is with School of Electrical and Electronic Engineering, University of Manchester, Manchester M13 9PL, United Kingdom.

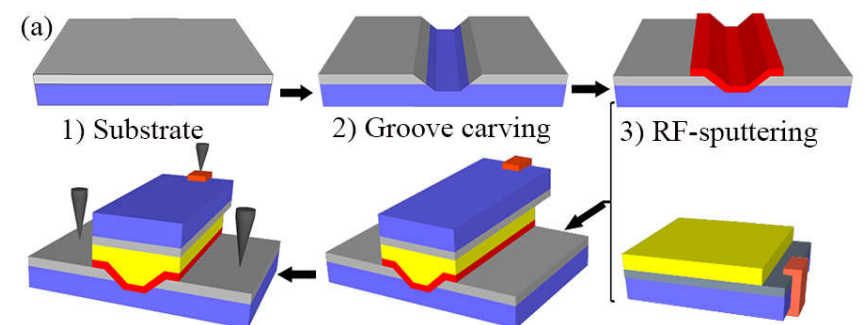

6) Measurement

5) Flip-chip bonding

4) Cut gate with PSSNa

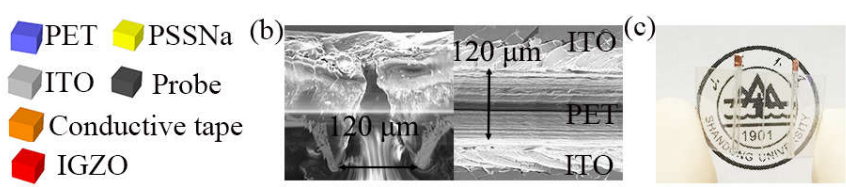

Fig. 1 (a) Illustration of the cross-sectional structure and the fabrication process of IGZO EDLTs. (b) Scanning electron microscopy of the groove. (c) Photographic image of the flexible IGZO EDLTs.

because of their high capacitance $(C)$ gate modulations [8]-[10]. In recent years, significant efforts have been focused on developing and exploring new electrolyte materials as well as novel processing methodologies to achieve two main objectives: enlarging $C$ to reduce the operation voltage, and lowering the processing temperature to be compatible with flexible plastic substrates in low cost. Solution processed ionic liquids or gels [11]-[18] and polymer electrolytes [19], [20] are frequently used as dielectric layers for fabrications of EDLTs. However, most of them require photolithography or printing to pattern the active area or electrolyte layer [12]-[14], [16]-[19]. Solid-state electrolytes, e.g. microporous $\mathrm{SiO}_{2}$, have been developed recently [21]-[25]. However, their fabrication processes, e.g. plasma-enhanced chemical-vapor deposition [21]-[23] and sputtering [24], [25], are usually time consuming (vacuum atmosphere, low deposition speed, etc.) compared to the solution processes. "Cut and stick" and "cut and bond" techniques have been developed for ion gel gated organic TFTs [26], [27]. However, only electrolyte layer and gate have been patterned in both techniques, and the source and drain contacts still need to be patterned by photolithography or shadow mask. Simple, low cost, and eco-friendly patterning techniques for preparing flexible EDLTs are highly desirable.

In this work, a "carving, cutting, and flip-chip bonding (CCFB)" technique is developed for polyelectrolyte gated flexible IGZO EDLTs. In this technique, the active area has been patterned by simple mechanical needle carving, and the gate has been patterned by cutting and flip-chip bonding with the electrolyte layer. The CCFB technique is simple, low cost, eco-friendly, easy to be applied in mass production, and can be used in flexible substrates. Based on the CCFB technique, high 
performance flexible IGZO EDLTs with a low operating voltage of $1 \mathrm{~V}$, a small threshold voltage $\left(V_{\text {th }}\right)$ of $0.51 \mathrm{~V}$, a very low subthreshold swing $(S S)$ of $76 \mathrm{mV} / \mathrm{dec}$, and a high current on/off ratio $\left(I_{\mathrm{on} / \mathrm{off}}\right)$ of about $1.4 \times 10^{7}$, have been realized.

\section{Experimental Details}

The fabrication process and the cross-sectional structure of the poly(styrenesulfonic acid sodium salt) (PSSNa) gated IGZO EDLTs are illustrated in Fig. 1(a). Flexible Polyethylene terephthalate (PET) covered by $200-\mathrm{nm}$ ITO was used as both substrates and electrodes (step 1 in Fig. 1(a)). A domestic cutting plotter (Kele, DC240) is used for both carving ITO-PET to form the channel area (step 2 in Fig. 1 (a)) and cutting ITO-PET for the gate electrodes (step 4 in Fig. 1(a)). Under such groove carving, the channel width $(\mathrm{W})$ and length $(\mathrm{L})$ of the EDLTs are $1 \mathrm{~cm}$ and $\sim 45-120 \mu \mathrm{m}$, respectively. The size of the cut ITO-PET gate chips is $1.0 \mathrm{~mm} \times 1.2 \mathrm{~cm}$. The carved ITO-PET with patterned source and drain electrodes and the cut ITO-PET gate chips were then cleaned in an ultrasonic bath of ethanol and DI water in succession, and then dried by nitrogen gas, to remove pollutants and the ITO residues produced by carving or cutting. Subsequently, IGZO layer with various thicknesses $(50,100,150,200 \mathrm{~nm})$ was deposited conformally into the carved grooves (step 3 in Fig. 1(a)) with a shadow mask by RF-sputtering at room temperature [28], for studying the influence of IGZO thickness on the device performance.

To prepare PSSNa polyelectrolyte gels, PSSNa (M.W. $\sim 70,000$, Alfa Aesar), D-sorbitol (Sigma-Aldrich), glycerol (Merck) and deionized water were mixed with a weight ratio of $40,10,10,40 \%$, respectively [29]. To facilitate electrical measurements, conductive copper tapes were pasted on one side of the gate chips from the ITO to the PET surface, as shown in Fig. 1(a) and (c). The polyelectrolyte was smeared onto these gate chips, as shown in step 4 in Fig. 1(a). Then, the $\mathrm{PSSNa} / \mathrm{ITO} / \mathrm{PET}$ chips were flipped and bonded onto the IGZO on the carved ITO-PET, as shown in step 5 in Fig. 1(a). The capacitance of the polyelectrolyte was measured by an LCR meter (Agilent E4980A). The electrical characteristics of the EDLTs were measured by source measure unit (Agilent B2902). The morphology of the groove was investigated using a scanning electron microscope (SEM, FEI Nova Nano450).
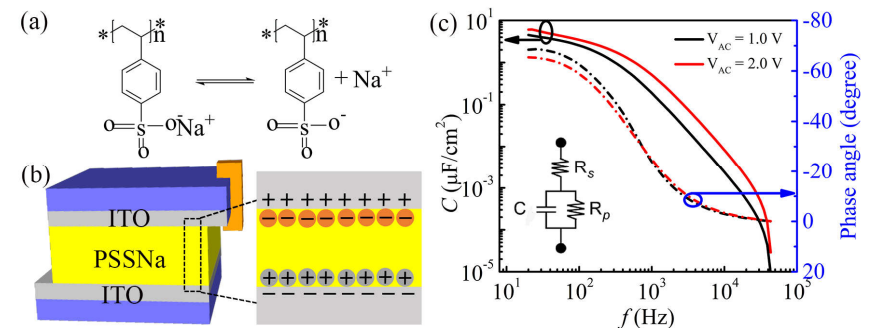

Fig. 2. (a) Chemical structure of PSSNa (left) and dissociated $\mathrm{PSS}^{-}$and $\mathrm{Na}^{+}$ (right) when a bias voltage is applied. (b) Schematic cross-section of the EDL capacitor. (c) Capacitance-frequency (short dash line) and phase angle-frequency (solid line) characteristics of the EDL capacitor. The inset represents the equivalent electric circuits.

\section{RESULTS AND DISCUSSION}

In order to study the capacitance of the EDL and its dependence on the frequency, we have fabricated an EDL capacitor with a structure of ITO/PSSNa polyelectrolyte/ITO. The chemical structure of PSSNa and the schematic cross-section of EDL capacitor are shown in Fig. 2(a) and (b). When an external voltage is applied, PSSNa dissociated into polyanions ( $\mathrm{PSS}^{-}$) and cations $\left(\mathrm{Na}^{+}\right)$[30]. The $\mathrm{Na}^{+}$ions move to the negatively biased electrode to form EDL, while polyanions stay close to the positively biased electrode as shown in Fig. 2(b). The EDLs are separated by only $\sim 1 \mathrm{~nm}$, which leads to extraordinary high $C$ and electric field [8], [12], [15]. The dependences of specific $C$ and phase-angle of the capacitor on frequency under different $\mathrm{AC}$ voltages are shown in Fig. 2(c). At low frequencies $(<100 \mathrm{~Hz})$, most of the $\mathrm{Na}^{+}$ions accumulate near the cathode/PSSNa polyelectrolyte interfaces, leading to the formation of a large EDL capacitance. At intermediate frequencies $(100 \mathrm{~Hz}<f<10 \mathrm{kHz})$, only a limited number of $\mathrm{Na}^{+}$ions close to the cathode can accumulate at the interfaces to form the EDL capacitance, and a large number of $\mathrm{Na}^{+}$ions drift in response to the electric filed and contribute to resistance. This leads to the dependent capacitance on frequency. At high frequencies (>10 kHz), almost all the $\mathrm{Na}^{+}$ ions drift in response to the electric field without accumulation, and thus the ITO/PSSNa/ITO works like a pure resistance. The capacitance reaches a maximum of $4.5 \mu \mathrm{F} / \mathrm{cm}^{2}$ at $20 \mathrm{~Hz}$. The phase angle settles at $-70^{\circ}$ instead of $-90^{\circ}$ at $20 \mathrm{~Hz}$. This is due to the voltage-induced tunneling current which contribute to a parallel resistance under the strong electric field of the EDL. The capacitance increases slightly with the increase of the amplitude of AC signal, due to the increase of the ionic conductivity under increased bias voltage [23]. The magnitude of the capacitance is essentially independent on thickness of the electrolyte layer [15], enabling ease of manufacturing via coating techniques such as smearing.

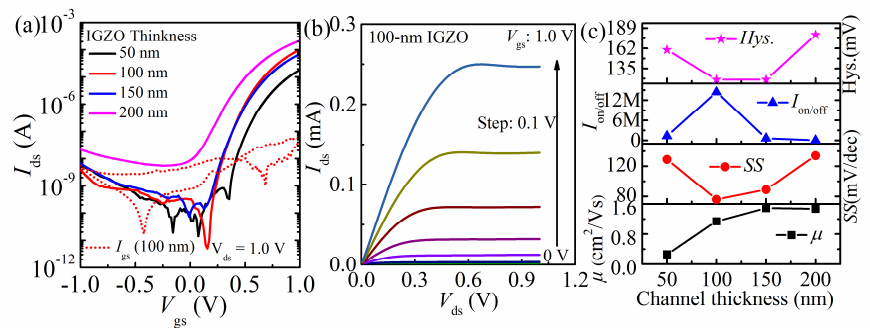

Fig. 3. (a) Transfer characteristics of the EDLTs with different IGZO thicknesses, and gate leakage current of IGZO EDLT with 100-nm IGZO. (b) Output characteristics of the EDLT with 100-nm IGZO. (c) The dependences of the $\mu, S S, I_{\text {on } / \text { off }}$ and hysteresis (Hys.) on IGZO thickness.

With the simple CCFB technique, EDLTs with various IGZO thicknesses $(50,100,150,200 \mathrm{~nm})$ were fabricated. It is interesting that despite the carving depth of the groove is even larger than $120 \mu \mathrm{m}$ and the carved surface is rough, as shown in Fig. 1(b), the EDLTs with IGZO layer of only $50 \sim 200 \mathrm{~nm}$ can realize low voltage $(\sim 1 \mathrm{~V})$ field effect carrier transport, as shown in Fig. 3(a), indicating the high applicability of the CCFB technique. The gate leakage current of these IGZO EDLTs is $\sim 10^{-8} \mathrm{~A}$, and is typical for electrolyte-gated TFTs [8], [22], [23]. The drain current $\left(I_{\mathrm{ds}}\right)$ of these EDLTs is more limited by the drift velocity of ions under higher frequencies. Thus, we used a low sweep rate of $18 \mathrm{mV} / \mathrm{s}$ for the transfer curve measurements. The output characteristic of the EDLT with 100-nm IGZO shown in Fig. 3(b), indicates the EDLT 
achieves large saturation current $\left(I_{\text {dsat }}=0.25 \mathrm{~mA}\right)$ under very low gate voltage, $V_{\mathrm{gs}}$, of $1 \mathrm{~V}$, reflecting strong gate modulation effect of the EDL capacitance. The linear region under low drain voltage, $V_{\mathrm{ds}}$, suggests ohmic contacts between the IGZO and ITO source/drain electrodes. The results illustrate that the IGZO EDLTs fabricated by CCFB technique have great potential for low-voltage and high on-current applications.

The influences of the IGZO thickness on the EDLTs performances are shown in Fig. 3 (a) and (c). The saturated mobility $(\mu)$ of these EDLTs are extracted from the transfer curve by using $I_{d s}=(W \mu \mathrm{C} / 2 L)\left(V_{g s}-V_{t h}\right)^{2}$ and taking an average $L$ of $120 \mu \mathrm{m}$, where $V_{\text {th }}$ is the threshold voltage. The mobility shows a clear increase with the IGZO thickness increases from 50 to $150 \mathrm{~nm}$, and then achieves saturation. It is suggested that deposition of the IGZO layer can reduce the surface roughness on the carved grooves, which can be called "filling the pothole". For the IGZO with the thicknesses of 50-100 nm, the thicker IGZO layer has a smoother top surface and lower trap state density at the channel interface. As the IGZO thickness increased to more than 100-150 nm, such "filling the pothole" effect is no longer dominant. SS first decreased to a minimum of only $76 \mathrm{mV} / \mathrm{dec}$ and then increased. $S S$ value is associated with the total trap density $\left(D_{\text {total }}\right)$, which is determined by the interface trap density $\left(D_{\mathrm{it}}\right)$, bulk trap density ( $\left.D_{\text {bulk }}\right)$, and the IGZO bottom surface trap density

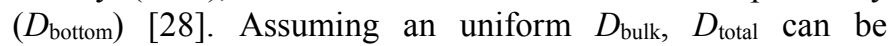
expressed as, $D_{\text {total }}=D_{\text {it }}+t D_{\text {bulk }}+D_{\text {bottom }}$ where $t$ is the thickness of the IGZO layer. $D_{\text {bottom }}$ can be assumed the same for all the EDLTs, as we carved the ITO-PET with the same mechanical parameters. $D_{\text {it }}$ should first decrease because the reduce of IGZO top surface roughness for $t$ increases from 50 to $100 \mathrm{~nm}$, and then reaches a saturation. As a result, the minimum $S S$ appears at the IGZO thickness of $100 \mathrm{~nm}$, indicating that the large roughness of back channel surface plays a predominant role for EDLTs with IGZO thinner than $100 \mathrm{~nm}$, whereas, the bulk traps became dominant for EDLTs with IGZO thicker than $100 \mathrm{~nm}$. Anticlockwise hysteresis with $V_{\text {th }}$ shifts of $0.12 \sim 0.18$ $\mathrm{V}$ are observed. The hysteresis shows a consistent trend with $S S$, supporting the traps effect in the channel as we discussed above. The EDLT with 100-nm IGZO shows the highest performance at a very low $V_{\mathrm{g}}$ of $-1 \sim+1 \mathrm{~V}$, with the maximum $I_{\mathrm{on} / \text { off }}$ of $1.4 \times$ $10^{7}$, smallest $S S$ of $76 \mathrm{mV} / \mathrm{dec}$, and high $\mu$ of $1.14 \mathrm{~cm}^{2} / \mathrm{Vs}$, indicating the CCFB technique is highly promising. In fact, the field effect property can be further improved by optimizing groove parameters including the size (depth, width, and length) and surface roughness, via optimizing the carving parameters such as the radius and materials of the carving needle, the carving force and speed, etc.

We have performed bias stress measurements for the optimized EDLT with 100-nm IGZO in order to study its stability under sustained bias. The pulse response measurement was carried out in dynamic stress tests, using a square-shaped pulses of $V_{\mathrm{gs}}=0$ to $1 \mathrm{~V}$ and $V_{\mathrm{ds}}=1 \mathrm{~V}$, as shown in Fig. 4(a). No obvious on current loss was observed after 40 testing cycles. The device also showed stable $I_{\mathrm{ds}}$ under the $V_{\mathrm{gs}}$ and $V_{\mathrm{ds}}$ both of $1 \mathrm{~V}$ sustained for 1000 seconds, as shown in Fig. 4(b). This suggests that there was no obvious chemical doping or chemical reaction in PSSNa/IGZO interface when the gate was biased [23].
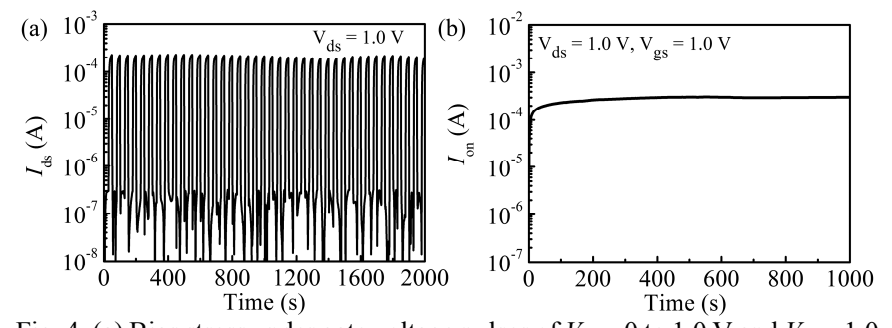

Fig. 4. (a) Bias stress under gate voltage pulses of $V_{\mathrm{gs}}=0$ to $1.0 \mathrm{~V}$ and $V_{\mathrm{ds}}=1.0$ V. (b) On current $\left(I_{\text {on }}\right)$ as a function of time.

In order to avoid the influence of interface traps caused by the substrate roughness on the stability of the EDLT, we used $\mathrm{SiO}_{2} / \mathrm{Si}$ as the substrate to study the effect of electrolyte on device stability, as shown in Fig. 5(a). The stability of the IGZO EDLT under negative gate bias stress (NGBS) and positive gate bias stress (PGBS) is shown in Fig. 5(b) and (c). Under negative gate bias, water can oxidize to protons and dioxygen, $2 \mathrm{H}_{2} \mathrm{O} \rightarrow 4 \mathrm{H}^{+}+\mathrm{O}_{2}+4 \mathrm{e}^{-}$[31]. These protons migrate under the applied electric field and accumulate at the gate electrode/PSSNa interface, which partially screens the applied gate voltage by creating an extra electric filed that compensates the gate electric field [31]. This causes that $V_{\text {th }}$ move towards the positive direction. A much smaller negative $V_{\text {th }}$ shift under the PGBS is observed, due to that the electric field induced generation of extra carriers in the channel layer [12]. The capping effect of the PSSNa and ITO/PET gate also contributes to the small PGBS.

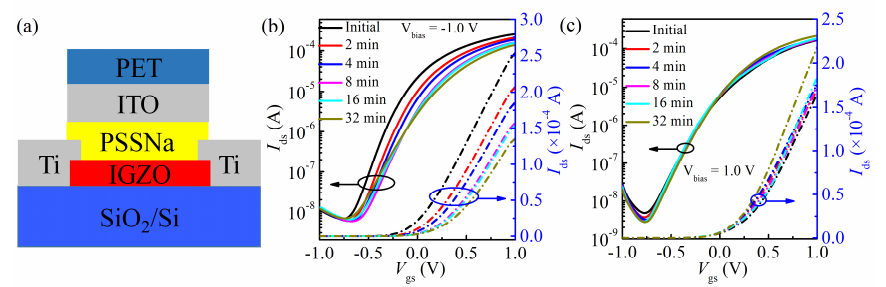

Fig. 5. Schematic of the cross-sectional IGZO EDLTs on $\mathrm{SiO}_{2} / \mathrm{Si}$ substrate (a). Effect of the $-1 \mathrm{~V}(\mathrm{~b})$ and the $1 \mathrm{~V}(\mathrm{c})$ gate bias stress of the IGZO EDLT under different stress durations at $V_{\mathrm{ds}}=1 \mathrm{~V}$.

\section{CONCLUSION}

We have developed a "carving, cutting, and flip-chip bonding" technique to fabricate polyelectrolyte gated EDLTs. The CCFB technique is simple, low cost, eco-friendly, and easy to be applied to mass production. With the CCFB technique, flexible IGZO EDLT exhibits a high performance with a high on/off ratio of $1.4 \times 10^{7}$, a nearly ideal subthreshold swing of $76 \mathrm{mV} / \mathrm{dec}$, a low operating voltage of less than $1 \mathrm{~V}$, high PGBS stability, which is comparable to or even better than those fabricated by photolithography or printing techniques.

\section{REFERENCES}

[1] S. Y. Lee, D. H. Kim, E. Chong, Y. W. Jeon, D. H. Kim, "Effect of channel thickness on density of states in amorphous InGaZnO thin film transistor", Appl. Phys. Lett., Vol. 98, No. 12, pp. 122105-1-122105-3, Mar. 2011, DOI: 10.1063/1.3570641

[2] H. Yabuta, M. Sano, K. Abe, T. Aiba, T. Den, H. Kumomi, H. Hosono, "High-mobility thin-film transistor with amorphous InGaZnO4 channel fabricated by room temperature rf-magnetron sputtering" Appl. Phys. Lett., Vol. 89, No. 11, pp. 112123-1-112123-3, Sept. 2006, DOI: $10.1063 / 1.2353811$ 
[3] K. Nomura, H. Ohta, A. Takagi, T. Kamiya, M. Hirano, H. Hosono, "Room-temperature fabrication of transparent flexible thin-film transistors using amorphous oxide semiconductors" Nature, Vol. 432, No. 7016, pp. 488-492. Nov. 2004, DOI: 10.1038/nature03090

[4] J. S. Park, T. W. Kim, D. Stryakhilev, J. S. Lee, S. G. An, Y. S. Pyo, H. K. Chung, "Flexible full color organic light-emitting diode display on polyimide plastic substrate driven by amorphous indium gallium zinc oxide thin-film transistors" Appl. Phys. Lett., Vol. 95, No. 1, pp. 013503-1-013503-3, Jul. 2009, DOI: 10.1063/1.3159832

[5] M. Nag, A. Bhoolokam, S. Smout, M. Willegems, R. Muller, K. Myny, P. Vicca, "Circuits and AMOLED display with self-aligned a-IGZO TFTs on polyimide foil" J. Soc. Inf. Display, Vol. 22, No. 10, pp. 509-517, Apr. 2015, DOI: $10.1002 /$ jsid.281

[6] M. Mativenga, D. Geng, B. Kim, J. Jang, "Fully transparent and rollable electronics", ACS Appl. Mater. Inter., Vol. 7, No. 3, pp. 1578-1585, Dec. 2015, DOI: 10.1021/am506937s

[7] I. D. Kim, Y. Choi, H. L. Tuller, "Low-voltage ZnO thin-film transistors with high-K Bi1.5Zn1.0Nb1.5O7 gate insulator for transparent and flexible electronics", Appl. Phys. Lett., Vol. 87, No. 4, pp. 043509-1-043509-3, Jul. 2005, DOI: 10.1063/1.1993762

[8] H. Du, X. Lin, Z. Xu, D. Chu, "Electric double-layer transistors: a review of recent progress", J. Mater. Sci., Vol. 50, No. 17, pp. 5641-5673, Sept. 2015, DOI: $10.1007 / \mathrm{s} 10853-015-9121-\mathrm{y}$

[9] T. Fujimoto, K. Awaga, "Electric-double-layer field-effect transistors with ionic liquids", Phys. Chem. Chem. Phys., Vol. 15, No. 23, pp. 8983-9006, Feb. 2013, DOI: 10.1039/C3CP50755F

[10] H. Yuan, H. Shimotani, J. Ye, S. Yoon, H. Aliah, A. Tsukazaki, Y. Iwasa, "Electrostatic and electrochemical nature of liquid-gated electric-double-layer transistors based on oxide semiconductors", J. Am. Chem. Soc., Vol. 132, No. 51, pp. 18402-18407, Dec. 2010, DOI: 10.1021/ja108912x

[11] C. J. Wan, Y. H. Liu, L. Q. Zhu, P. Feng, Y. Shi, Q. Wan, "Short-term synaptic plasticity regulation in solution-gated indium-gallium-zinc-oxide electric-double-layer transistors", ACS Appl. Mater. Inter., Vol. 8, No. 15, pp. 9762-9768, Mar. 2016, DOI: 10.1021/acsami.5b12726

[12] P. R. Pudasaini, J. H. Noh, A. T. Wong, O. S. Ovchinnikova, A. V. Haglund, S. Dai, P. D. Rack, "Ionic Liquid Activation of Amorphous Metal-Oxide Semiconductors for Flexible Transparent Electronic Devices", Adv. Funct. Mater., Vol. 26, No. 17, pp. 2820-2825, Feb. 2016, DOI: $10.1002 /$ adfm.201505274

[13] J. M. Black, J. Come, S. Bi, M. Zhu, W. Zhao, A. T. Wong, S. Dai, "Role of Electrical Double Layer Structure in Ionic Liquid Gated Devices", ACS Appl. Mater. Inter., Vol. 9, No. 46, pp. 40949-40958, Oct. 2017, DOI: $10.1021 /$ acsami.7b11044

[14] K. Hong, S. H. Kim, K. H. Lee, C. D. Frisbie, "Printed, sub-2V ZnO electrolyte gated transistors and inverters on plastic", Adv. Mater., Vol. 25, No. 25, pp. 3413-3418, Mar. 2013, DOI: 10.1002/adma.201300211

[15] S. H. Kim, K. Hong, W. Xie, K. H. Lee, S. Zhang, T. P. Lodge, C. D. Frisbie, "Electrolyte-Gated Transistors for Organic and Printed Electronics", Adv. Mater., Vol. 25, No. 13, pp. 1822-1846, Dec. 2012, DOI: 10.1002 /adma.201202790

[16] M. S. Chae, J. H. Park, H. W. Son, K. S. Hwang, T. G. Kim, "IGZO-based electrolyte-gated field-effect transistor for in situ biological sensing platform", Sensor. Actuat. B-Chem., Vol. 262, No. 1, pp. 876-883, Jun. 2018, DOI: 10.1016/j.snb.2018.02.090

[17] N. Kumar, J. Kumar, S. Panda, "Back-channel electrolyte-gated a-IGZO dual-gate thin-film transistor for enhancement of $\mathrm{pH}$ sensitivity over nernst limit", IEEE Electron Device Lett., Vol. 37, No. 4, pp. 500-503, Mar. 2016, DOI: 10.1109/LED.2016.2536359

[18] S. Park, S. Lee, C. H. Kim, I. Lee, W. J. Lee, S. Kim, M. H. Yoon, "Sub-0.5 V Highly stable aqueous salt gated metal oxide electronics", Sci. Rep., Vol. 5, Aug. 2015, DOI: 10.1038/srep13088

[19] N. Kumar, J. Kumar, S. Panda, "Enhanced pH sensitivity over the Nernst limit of electrolyte gated a-IGZO thin film transistor using branched polyethylenimine", RSC Adv., Vol. 6, No. 13, pp. 10810-10815, Jan. 2016, DOI: $10.1039 /$ C5RA26409J

[20] S. H. Chen, H. C. Liu, C. Y. Lee, J. Y. Gan, H. W. Zan, J. C. Hwang, P. C. Lyu, "High performance electric-double-layer amorphous IGZO thin-film transistors gated with hydrated bovine serum albumin protein", Org. Electron., Vol. 24, pp. 200-204, Sept. 2015, DOI: 10.1016/j.orgel.2015.05.046

[21] J. Zhou, N. Liu, L. Zhu, Y. Shi, Q. Wan, "Energy-efficient artificial synapses based on flexible IGZO electric-double-layer transistors", IEEE
Electron Device Lett., Vol. 36, No. 2, pp. 198-200, Dec. 2014, DOI: 10.1109/LED.2014.2381631

[22] J. Jiang, Q. Wan, J. Sun, A. Lu, "Ultralow-voltage transparent electric-double-layer thin-film transistors processed at room-temperature", Appl. phys. Lett., Vol. 95, No. 15, pp. 152114-1-152114-3, Oct. 2009, DOI: 10.1063/1.3251782

[23] J. Sun, J. Jiang, A. Lu, Q. Wan, “One-Volt Oxide Thin-Film Transistors on Paper Substrates Gated by $\mathrm{SiO}_{2}$-Based Solid Electrolyte with Controllable Operation Modes", IEEE T. Electron. Dev., Vol. 57, No. 9, pp. 2258-2263, Sept. 2010, DOI: 10.1109/TED.2010.2052168

[24] W. Cai, J. Zhang, J. Wilson, X. Ma, H. Wang, X. Zhang, A. Song, "Oxide-Based Electric-Double-Layer Thin-Film Transistors on a Flexible Substrate", IEEE Electron Device Lett., Vol. 38, No. 12 pp. 1680-1683, Dec. 2017, DOI: 10.1109/LED.2017.2768822

[25] X. Ma, J. Zhang, W. Cai, H. Wang, J. Wilson, Q. Wang, A. Song, “A sputtered silicon oxide electrolyte for high-performance thin-film transistors", Sci. Rep., Vol. 7, Apr. 2017, DOI: 10.1038/s41598-017-00939-6

[26] K. H. Lee, M. S. Kang, S. Zhang, Y. Gu, T. P. Lodge, C. D. Frisbie, "Cut and stick" rubbery ion gels as high capacitance gate dielectrics", $A d v$. Mater., Vol. 24, No. 32, pp. 4457-4462, Jul. 2012, DOI: 10.1002/adma.201200950

[27] X. Shao, B. Bao, J. Zhao, W. Tang, S. Wang, X. Guo, "Low-Voltage Large-Current Ion Gel Gated Polymer Transistors Fabricated by a "Cut and Bond" Process", ACS Appl. Mater. Inter., Vol. 7, No. 8, pp. 4759-4762, Jan. 2015, DOI: 10.1021/am508487z

[28] B. Zhang, H. Li, X. Zhang, Y. Luo, Q. Wang, A. Song, "Performance regeneration of InGaZnO transistors with ultra-thin channels", Appl. Phys. Lett., Vol. 106, No. 9, pp. 093506-1-093506-5. Mar. 2015, DOI: $10.1063 / 1.4914296$

[29] P. A. Ersman, D. Nilsson, J. Kawahara, G. Gustafsson, M. Berggren, "Fast-switching all-printed organic electrochemical transistors", Org. Electron., Vol. 14, No. 5, pp. 1276-1280, May. 2013, DOI: 10.1016/j.orgel.2013.02.027

[30] E. Said, X. Crispin, L. Herlogsson, S. Elhag, N. D. Robinson, M. Berggren, "Polymer field-effect transistor gated via a poly (styrenesulfonic acid) thin film", Appl. Phys. Lett., Vol. 89, No. 14, pp. 143507-1-143507-3, Oct. 2006, DOI: 10.1063/1.2358315

[31] H. Sinno, S. Fabiano, X. Crispin, M. Berggren, I. Engquist, "Bias stress effect in polyelectrolyte-gated organic field-effect transistors" Appl. Phys. Lett., Vol. 102, No. 11, p. 50, Mar. 2013, DOI: 10.1063/1.4798512 